\title{
Utilizing Terahertz Band for Local and Personal Area Wireless Communication Systems
}

\author{
Turker Yilmaz Ozgur B. Akan \\ Next-generation and Wireless Communications Laboratory (NWCL) \\ Department of Electrical and Electronics Engineering \\ Koc University, Istanbul, Turkey \\ Email: \{turkeryilmaz, akan\}@ku.edu.tr
}

\begin{abstract}
User demand on wireless communications is growing every day, however frequency spectrum is a scarce source that cannot infinitely supply the ever-increasing communication demand. Most of the research activities addressing this problem have traditionally been focused on spectral efficiency improvement and signalling overhead minimization efforts, therefore improvement in these domains is tougher than ever. The other main method for throughput enhancement is increasing the operation frequency, and towards this end, the utilization of terahertz band for the next-generation of wireless communications systems is investigated in this paper. Following a general overview, an indoor computer simulation is set up to reveal both the feasibility and advantages of $\mathrm{THz}$ band compared to the currently used frequency spectrum. The results show that, peak data rates on the order of $10 \mathrm{~Gb} / \mathrm{s}$ is possible over low-THz band; however, there are hindering issues such as coverage area and low-cost device availability.
\end{abstract}

Index Terms-Future wireless communication, millimetre-wave communication, submillimeter-wave communication, numerical simulation.

\section{INTRODUCTION}

Humankind is going through the most connected era of its history due to the unending advancements in transportation and communications. Correspondingly, both the number of wireless connections and traffic volume per user is constantly increasing. The number of global mobile devices and connections grew to 7 billion in 2013 and this figure is expected to increase to 10.2 billion by the end of 2018, corresponding to a compound annual growth rate (CAGR) of $8 \%$ [1]. Global mobile data traffic also rose by $81 \%$ in 2013 and predicted to grow 11-fold within 5 years with a CAGR of $61 \%$ [1].

International Telecommunication Union (ITU) Radiocommunication Sector (ITU-R) named third generation mobile telecommunication systems as International Mobile Telecommunications-2000 (IMT-2000) and fourth generation (4G) as IMT-Advanced and the radio interface objectives of these are defined in the reports ITU-R M.1034-1 [2] and ITUR M.2134 [3], respectively. Composed in 1997 to a different telecommunications environment where network integration was not existent, six service types, namely speech, audio, data, text, image and video, were defined for IMT-2000 with maximum data rate provisionally identified to be $20 \mathrm{Mb} / \mathrm{s}$ for data and video services and available for single cells. ITU-R M.2134, on the other hand, declares minimum downlink peak spectral efficiency to be $15 \mathrm{~b} / \mathrm{s} / \mathrm{Hz}$ and as bandwidths up to
$100 \mathrm{MHz}$ are encouraged for utilization, peak data rates of $1500 \mathrm{Mb} / \mathrm{s}$ are articulated for 4G systems.

This continuously increasing demand for wireless communications requires major throughput and capacity enhancements, the main methods for which are widely known: Increasing the spectral efficiency or operation bandwidth, or decreasing the signalling overhead. Spectral efficiency is the most explored, and thus, the most developed of the three, leading to smallest amount of return for any new research effort invested. Having an indirect effect, reduction in signalling overhead also cannot generate major improvements, leaving the operation bandwidth as the method to pursue. However, apart from the industry resistance towards changing the already established communications spectrum, there are also not many suitable frequency bands available for general communications purposes. Still, due to the unavoidable necessity, formal standardization activities on 60 gigahertz $(\mathrm{GHz})$ industrial, scientific and medical (ISM) radio band officially began in March 2005 with the formation of IEEE 802.15 Task Group $3 \mathrm{c}$, and the first widespread commercial products under WiFi Alliance's certification are expected to be available for purchase in 2015.

This paper concentrates on increasing the carrier frequency, and thus, using more bandwidth to counter the higher data rate and volume demands of wireless communication networks. This work develops the analyses provided in [4] and, to the best of authors' knowledge, is the first study presenting realistic transmission simulations above $350 \mathrm{GHz}$, revealing the upper limit of realistic terahertz $(\mathrm{THz})$ communications. With these aims, Section II sets up the simulations, Section III provides the performance evaluation and the paper concludes with Section IV.

\section{INDOOR SIMULATIONS}

Keeping in mind the narrow coverage submillimeter-wave (submm-wave) communication can offer, the author's laboratory and the corridor in front of the room is selected for simulation, the details of which are available in [4]. Due to the unavailability of transmission properties of many building materials in $\mathrm{THz}$ band, the walls are assumed to be clay brick (CB) and the door made of medium-density fiberboard (MDF).

$\mathrm{THz}$ simulation parameters are also further constrained by the available literature on the subject. Although research on 
$\mathrm{THz}$ band devices is on a sharp rise, for a technique to be suitable for adoption in wireless communication systems it primarily has to be inexpensive. At present only devices of silicon and complementary metaloxidesemiconductor origin possess that requirement and this further narrows published solutions. To the best of authors' knowledge, a tunable signal source within low-THz range is able to radiate $-1.19 \mathrm{dBm}$ for output power at most [5]; therefore, transmit power for $\mathrm{THz}$ simulations is taken as $0 \mathrm{dBm}$.

Due to the additional free-space loss on the order of 40 $\mathrm{dB}$ occurring at low- $\mathrm{THz}$ band, a compensation has to be generated for viable $\mathrm{THz}$ communication links. Since transmitting power is decided upon, only antenna gains are left for solution. In the extreme case of lens use, leaving the half-power beamwidth consequence aside, a gain of $35.6 \mathrm{dBi}$ is reported [6]; however, EM radiation power densities are regulated by respective authorities. For the case of Europe, European Telecommunications Standards Institute limit the mean equivalent isotropically radiated power (EIRP) to 20 $\mathrm{dBm}$ for the $2.4 \mathrm{GHz}$ ISM band [7] and 20, 23, 27 and 30 $\mathrm{dBm}$ for different parts of the $5 \mathrm{GHz}$ ISM band [8]. For the simulations, an EIRP of $23 \mathrm{dBm}$ is assumed, thus setting the antenna gains on both ends at $23 \mathrm{dBi}$.

Four simulations are carried out, calculating the received power and signal-to-noise ratio (SNR). All simulations use the same parameters and environment, except for the increasing carrier frequency and the proportional noise bandwidth. Due to the available material properties data [9], simulation frequencies are set at 100, 350, 500 and $700 \mathrm{GHz}$. Taking a comparative approach to the communications standards on 60 $\mathrm{GHz}$, which set channel spacing as $2160 \mathrm{MHz}$, bandwidths are selected to be 3.6, 12.6, 18 and $25.2 \mathrm{GHz}$, respectively. Noise figures and system margin are again chosen according to [4] and ground-level standard temperature and water-vapour density quantities are employed from [10].

Since there are no comprehensive channel or path loss models available for $\mathrm{THz}$ band, ray tracing technique is employed [11]. Received power $\left(P_{r}\right)$ and SNR for line-ofsight (LoS) situations are calculated, with $d$ being distance in metres and $f_{c}$ carrier frequency in $\mathrm{Hz}$, using

$$
\begin{aligned}
P_{r} & =\left(P_{t}+G_{t}+G_{r}\right)-\left(\frac{20 \log (4 \pi d)}{\left(C_{0} / f_{c}\right)}\right)-\left(\gamma d 10^{-3}\right) \\
\mathrm{SNR} & =P_{r}-\left(N_{0}+10 \log (B)+\mathrm{NF}+\mathrm{SM}\right)
\end{aligned}
$$

where $P_{t}$ is transmitting power, $G_{t}$ and $G_{r}$ are transmitter (TX) and receiver (RX) antenna gains, respectively, $N_{0}$ is noise spectral density and $B$ is the bandwidth employed. $\gamma$ represents specific gaseous attenuation in $\mathrm{dB} / \mathrm{km}$, therefore, the $10^{-3}$ term added to convert $d$ into km, and Fig.1 illustrates the change in this attenuation with frequency for standard atmospheric conditions of $15{ }^{\circ} \mathrm{C}, 1013 \mathrm{hPa}$ and varying water vapour densities of $0,7.5$ and $15 \mathrm{~g} / \mathrm{m}^{3}$. The middle part of (1) is the Friss equation with $C_{0}$ being speed of light in free space in $\mathrm{m} / \mathrm{s}$ and for non-line-of-sight $(\mathrm{NLoS})$ an additional absorption loss is subtracted from (1), which is calculated using Lambert-Beer's attenuation law as detailed

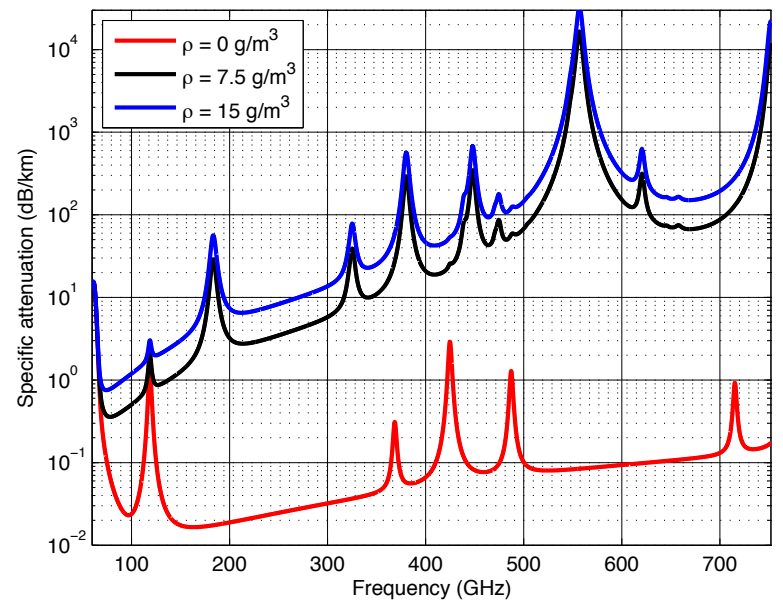

Fig. 1. Attenuation due to atmospheric gases, calculated between 59.755 and $752.048 \mathrm{GHz}$, and for water vapour densities of $0,7.5$ and $15 \mathrm{~g} / \mathrm{m}^{3}$.

TABLE I

\begin{tabular}{|c|c|c|c|c|}
\hline Quantity & $100 \mathrm{G}$ & $350 \mathrm{G}$ & $500 \mathrm{G}$ & $700 \mathrm{G}$ \\
\hline $\begin{array}{l}\text { Transmitting power } \\
T X \text { and RX antenna gain }\end{array}$ & & \multicolumn{2}{|c|}{$\begin{array}{l}0 \mathrm{dBm} \\
23 \mathrm{dBi}\end{array}$} & \\
\hline $\begin{array}{l}\text { BS noise figure } \\
\text { UE noise figure } \\
\text { System margin }\end{array}$ & & \multicolumn{2}{|c|}{$\begin{array}{l}7 \\
5\end{array}$} & \\
\hline $\begin{array}{l}\text { Noise bandwidth }(\mathrm{GHz}) \\
\alpha_{\mathrm{CB}}\left(\mathrm{m}^{-1}\right) \\
\alpha_{\mathrm{MDF}}\left(\mathrm{m}^{-1}\right)\end{array}$ & $\begin{array}{c}3.6 \\
86 \\
116\end{array}$ & $\begin{array}{l}12.6 \\
482 \\
461\end{array}$ & $\begin{array}{c}18 \\
916 \\
779\end{array}$ & $\begin{array}{l}25.2 \\
1500 \\
1310\end{array}$ \\
\hline
\end{tabular}

SIMULATION PARAMETERS

in [4]. Absorption coefficients $(\alpha)$ through walls and door are assumed to be constant and obtained from the respective values of $\mathrm{CB}$ and MDF as reported in [9]. The only coefficient that is not available, $\alpha_{\mathrm{CB}}$ at $700 \mathrm{GHz}$, is interpolated to $1500 \mathrm{~m}^{-1}$ using the respective graph illustrated in [9]. The simulation parameters overall are listed in Table I.

\section{RESULTS AND DISCUSSION}

The calculations are performed for each of the $1 \mathrm{~cm}^{3}$ volume within the described environment and results are provided for a representative height of $1 \mathrm{~m}$. A summary of extrema of all simulations are presented in Table II, where TN denotes thermal noise.

\section{A. $100 \mathrm{GHz}$}

Received power and SNR results of $100 \mathrm{GHz}$ simulation are provided in Fig.2. Since there is a single source available, power, as expected, dissipates in a uniformly circular manner within the laboratory LoS region. The corridor, however, does not adhere to this explanation and requires the observation of SNR outcome which is illustrated in Fig.2b.

The first point to notice in Fig.2b is the trapezoid shape, which is also present but unnoticeable in Fig.2a due to very broad colour range. The reasons for this formation are the 
TABLE II

SiMULATION RESULTS

\begin{tabular}{|c|c|c|c|c|c|c|c|c|c|}
\hline \multirow{2}{*}{ Quantity } & & \multicolumn{4}{|c|}{ Laboratory } & \multicolumn{4}{|c|}{ Corridor } \\
\hline & & $100 \mathrm{G}$ & $350 \mathrm{G}$ & $500 \mathrm{G}$ & $700 \mathrm{G}$ & $100 \mathrm{G}$ & $350 \mathrm{G}$ & $500 \mathrm{G}$ & $700 \mathrm{G}$ \\
\hline \multirow{2}{*}{ Received power $(\mu \mathrm{W})$} & $\min$ & 0.0299 & 0.0024 & 0.001 & 0.0005 & $5.72 \times 10^{-13}$ & $1.83 \times 10^{-63}$ & $5.84 \times 10^{-118}$ & $4.06 \times 10^{-191}$ \\
\hline & $\max$ & 0.4435 & 0.036 & 0.0171 & 0.0087 & 0.0005 & $2.41 \times 10^{-11}$ & $2.19 \times 10^{-17}$ & $3.37 \times 10^{-27}$ \\
\hline \multirow{2}{*}{$S N R(\mathrm{~dB})$} & $\min$ & 16.199 & -0.214 & -5.3552 & -9.9078 & -90.981 & $\ll \mathrm{TN}$ & $\ll \mathrm{TN}$ & $\ll \mathrm{TN}$ \\
\hline & $\max$ & 27.911 & 11.566 & 6.7901 & 2.3624 & -2.0154 & -80.182 & -142.14 & -241.73 \\
\hline
\end{tabular}

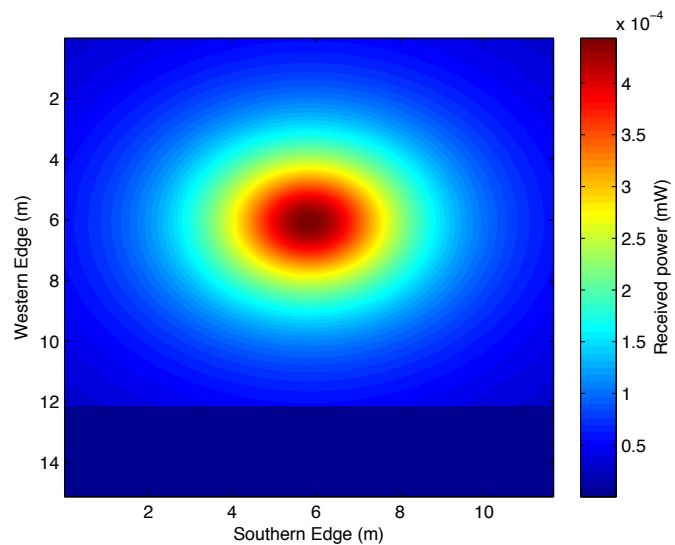

(a)

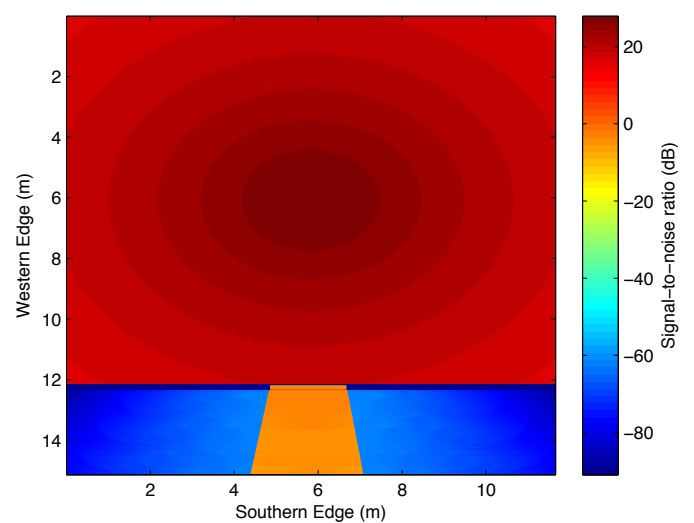

(b)

Fig. 2. (a) Received power and (b) SNR performance of $100 \mathrm{GHz}$ at indoor simulation for $h=1 \mathrm{~m}$.

differences between the thicknesses and absorption coefficients of $\mathrm{CB}$ and MDF and the inclined electromagnetic (EM) waves that penetrate through the door to reach the $h=1 \mathrm{~m}$ plane. The SNR values within this semi-permeable region vary between $2 \mathrm{~dB}$ and $-6 \mathrm{~dB}$, whereas it is below $-60 \mathrm{~dB}$ over the remainder of the hall.

The LoS laboratory area benefits from SNR values in the range of $16 \mathrm{~dB}$ to $28 \mathrm{~dB}$. As successful wireless transmission with 16-ary quadrature amplitude modulation (16-QAM) requires an SNR of $14.9 \mathrm{~dB}$ for a bit error ratio (BER) of $10^{-6}$, within the laboratory a theoretical total throughput of $14.4 \mathrm{~Gb} / \mathrm{s}$, before signalling overhead, is possible. The requirement for an uncompressed high-definition video traffic utilizing 60 frames/s is just short of $2.8 \mathrm{~Gb} / \mathrm{s}$. Therefore, this setting is capable of supporting any wireless display applications within the range limits of wireless personal area networks (WPANs). Cables can also be replaced with this type of wireless transceivers since even the latest high-definition multimedia interface specification, version 2.0, sets maximum throughput per channel at $6 \mathrm{~Gb} / \mathrm{s}$ [12]. Thus, all personal area network applications, ranging from local file transfer to docking functionalities, can theoretically be replicated via a carrier at $100 \mathrm{GHz}$ frequency.

The NLoS corridor area, on the other hand, is incapable of supporting high data rate transmissions according to the performed simulation. A $100 \mathrm{GHz}$ carrier traversing $1 \mathrm{~cm}$ thick $\mathrm{CB}$ loses $3.735 \mathrm{~dB}$ of its power, whereas this value is 5.038
$\mathrm{dB}$ for MDF. Considering simulated EM waves propagated through in excess of $20 \mathrm{~cm}$ of $\mathrm{CB}$ or $4 \mathrm{~cm}$ of MDF to reach the corridor, the proportionally increasing absorption losses emerge as the main reason for the stated inability. However, the two region outcome suggests the possibility of handover, which is critical for uninterrupted wireless communications. The SNR values within the semi-permeable region behind the MDF door are high enough to assist transfer of communication links from one access point (AP) to another. Hence, this deduction can be utilized to even realize the next-generation of mobile telecommunication systems indoors at frequencies in the neighbourhood of $100 \mathrm{GHz}$, by constructing buildings using $\mathrm{THz}$-friendly materials and implementing access networks, which will address the specific challenges of submmwave communication.

\section{B. $350 \mathrm{GHz}$}

Fig.3 illustrates the received power and SNR outcome of the $350 \mathrm{GHz}$ simulation. The received power performance given in Fig.3a directly replicates the $100 \mathrm{GHz}$ result, except for the reduced levels. In fact, this is also the case for the 500 $\mathrm{GHz}$ and $700 \mathrm{GHz}$ simulations, for which reason the power outcomes of those are not provided.

The more significant SNR results are provided in Fig.3b. The first difference compared to the $100 \mathrm{GHz}$ case is in visualization. Because the lower end of the received power is very small - in fact practically undetectable, the corresponding SNR values are also very small. This widens the colour range 


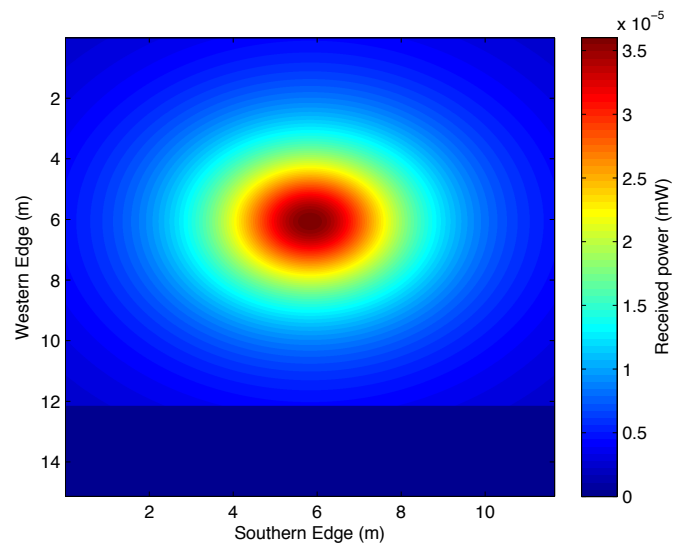

(a)

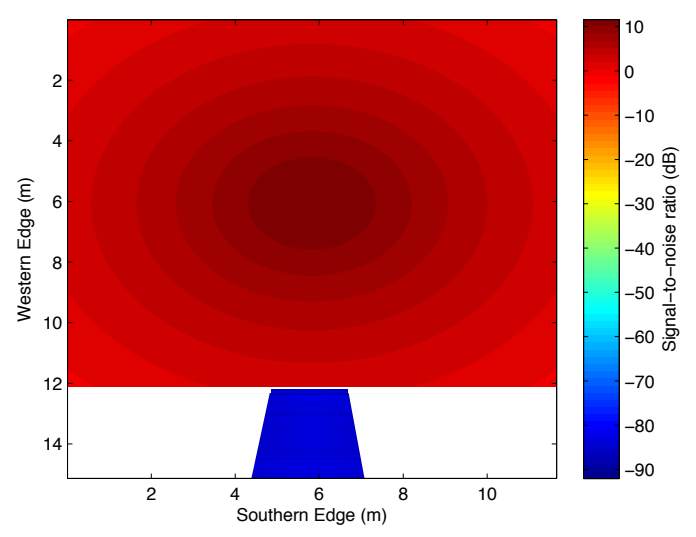

(b)

Fig. 3. (a) Received power and (b) SNR performance of $350 \mathrm{GHz}$ at indoor simulation for $h=1 \mathrm{~m}$.

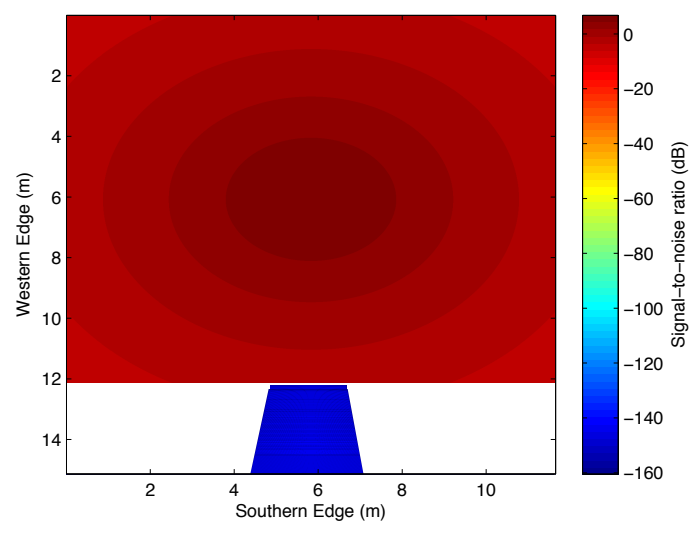

(a)

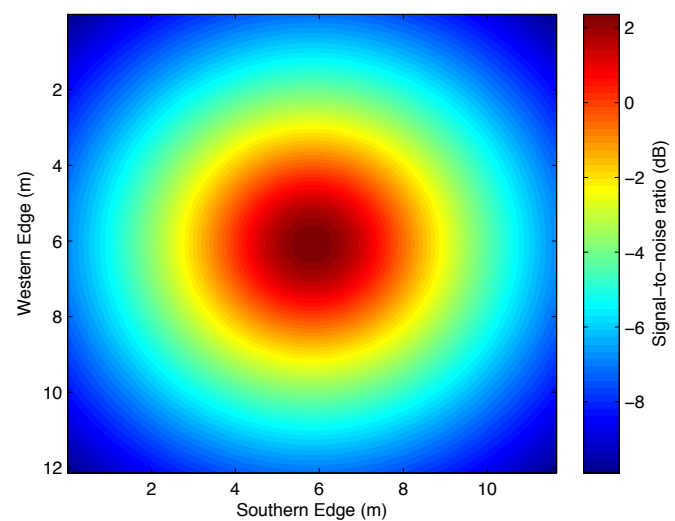

(b)

Fig. 4. SNR performance of (a) $500 \mathrm{GHz}$ and (b) $700 \mathrm{GHz}$ at indoor simulation for $h=1 \mathrm{~m}$.

to such extent that figure becomes incomprehensible. To make the result plainer, the wall and the corridor area accessible through wall are removed from the SNR performance plots of $350 \mathrm{GHz}$ and $500 \mathrm{GHz}$ simulations.

The SNR results of the laboratory vary between $-0.2 \mathrm{~dB}$ and $11.6 \mathrm{~dB}$. While $0 \mathrm{~dB}$ does not provide a hospitable environment for high data rate communications, $10.78 \mathrm{~dB}$ is enough for a BER of $10^{-6}$ using 4-QAM. Since this only halves the spectral efficiency while the simulation bandwidth is tripled compared to the $100 \mathrm{GHz}$ case, all the applications listed before are also feasible for $350 \mathrm{GHz}$ carrier. The shortcoming is the reduced coverage due to the higher free space path (FSPL) and atmospheric losses. For the same distance, EM waves lose $10.88 \mathrm{~dB}$ more at $350 \mathrm{GHz}$ because of FSPL, and the specific attenuation due to atmospheric gases are 10.941 and $0.503 \mathrm{~dB} / \mathrm{km}$ for 350 and $100 \mathrm{GHz}$, respectively.

The values behind the door in the hall are between $80 \mathrm{~dB}$ and $-92 \mathrm{~dB}$. The $\mathrm{TN}$ is $-73 \mathrm{~dB}$ for the $350 \mathrm{GHz}$ simulation parameters, so in this case it is not possible to provide any transmission into the hall area. Absorption is again the main reason, since through $1 \mathrm{~cm}$ of $\mathrm{CB}$ and $\mathrm{MDF}$ the losses are $20.933 \mathrm{~dB}$ and $20.021 \mathrm{~dB}$, respectively. Therefore, to realize indoor wireless communications at $350 \mathrm{GHz}$ either materials generating lesser absorption losses should be used, or a network architecture with at least one AP per room should be adopted.

\section{C. $500 \mathrm{GHz}$ and $700 \mathrm{GHz}$}

For the remaining simulations only the SNR outputs are given in Fig.4. While the values of the semi-permeable region behind the door are available for $500 \mathrm{GHz}$, since the highest NLoS SNR output is calculated as $-241.73 \mathrm{~dB}$ in the $700 \mathrm{GHz}$ case, just the results within the LoS limits of the laboratory are demonstrated in Fig.4b.

The LoS SNR results vary between $-5.4 \mathrm{~dB}$ and $6.8 \mathrm{~dB}$ in the $500 \mathrm{GHz}$ simulation. The upper limit is adequate to sustain successful wireless communications using binary phase-shift keying (PSK) with a BER of $10^{-3}$, or quadrature PSK or 4QAM with a BER of $2 \times 10^{-3}$. Comparison of these results 
with the $350 \mathrm{GHz}$ case illustrates that further carrier frequency increase causes worse maintainable BERs, even shorter coverage range and no improvement on spectral efficiency. While higher bandwidth would assist short-range data rate intensive applications like rapid sync-n-go file transfer [13], the considerably worse BER is preventative towards wireless display implementations at $500 \mathrm{GHz}$.

LoS SNR values of $700 \mathrm{GHz}$ simulation resulted between $-9.9 \mathrm{~dB}$ and $2.4 \mathrm{~dB}$. These levels clearly cannot support a communication link but rather be useful for handover. Moreover, NLoS SNR values of 500 and $700 \mathrm{GHz}$ are below $-142.14 \mathrm{~dB}$ and $-241.73 \mathrm{~dB}$, respectively, both which are remarkably lower than TN. Innovative developments are still required in multiple different domains of wireless communications for these test settings to be operational.

\section{CONCLUSION}

In this paper, the use of $\mathrm{THz}$ band for wireless communication systems is investigated. Subsequent to a general introduction on the current state of wireless and mobile communication systems, the realistic simulation environment of [4] is extended across $700 \mathrm{GHz}$. According to the results, transmission windows up to $500 \mathrm{GHz}$ are capable of supporting WPANs, which can theoretically provide peak data rates between $10 \mathrm{~Gb} / \mathrm{s}$ and $20 \mathrm{~Gb} / \mathrm{s}$. Severe material absorption on the higher frequencies is the principal reason against coverage expansion. Towards that end, indoor access network architectures featuring multiple APs that support a handover mechanism and building materials with workable absorption coefficients should be investigated.

\section{ACKNOWLEDGEMENT}

This work was supported in part by the Scientific and Technological Research Council of Turkey (TUBITAK) under grant \#113E962.

\section{REFERENCES}

[1] "Cisco Visual Networking Index: Global Mobile Data Traffic Forecast Update, 2013-2018," Cisco Systems, Inc., Report, 2014.

[2] "Report ITU-R M.1034-1: Requirements for the radio interface(s) for International Mobile Telecommunications-2000 (IMT-2000)," ITU-R Recommendations and Reports, ITU, Geneva, Switzerland, 1997.

[3] "Report ITU-R M.2134: Requirements related to technical performance for IMT-Advanced radio interface(s)," ITU-R Recommendations and Reports, ITU, Geneva, Switzerland, 2008.

[4] Yilmaz, Turker and Fadel, Etimad and Akan, Ozgur B., "Employing 60 GHz ISM band for 5G wireless communications," in Communications and Networking (BlackSeaCom), 2014 IEEE International Black Sea Conference on, Conference Proceedings, pp. 77-82.

[5] Tousi, Y. M. and Momeni, O. and Afshari, E., "A Novel CMOS High-Power Terahertz VCO Based on Coupled Oscillators: Theory and Implementation," Solid-State Circuits, IEEE Journal of, vol. 47, no. 12, pp. 3032-3042, 2012

[6] Priebe, S. and Jacob, M. and Kurner, T., "The impact of antenna directivities on $\mathrm{THz}$ indoor channel characteristics," in Antennas and Propagation (EUCAP), 2012 6th European Conference on, Conference Proceedings, pp. 478-482.

[7] ETSI, "EN 300328 V1.8.1 (2012-06)," Electromagnetic compatibility and Radio spectrum Matters (ERM); Wideband transmission systems; Data transmission equipment operating in the 2,4 GHz ISM band and using wide band modulation techniques; Harmonized EN covering essential requirements of article 3.2 of the R\&TTE Directive, 2012
[8] — "EN 301893 V.1.7.1 (2012-06)," Broadband Radio Access Networks (BRAN); $5 \mathrm{GHz}$ high performance RLAN; Harmonized EN covering the essential requirements of article 3.2 of the R\&TTE Directive, 2012.

[9] Piesiewicz, R. and Jansen, C. and Wietzke, S. and Mittleman, D. and Koch, M. and Kurner, T., "Properties of Building and Plastic Materials in the THz Range," International Journal of Infrared and Millimeter Waves, vol. 28, no. 5, pp. 363-371, 2007.

[10] "Recommendation ITU-R P.835-5: Reference standard atmospheres," ITU-R Recommendations, P Series Fasicle, ITU, Geneva, Switzerland, 2012.

[11] Sarkar, T. K. and Zhong, Ji and Kyungjung, Kim and Medouri, A. and Salazar-Palma, M., "A survey of various propagation models for mobile communication," Antennas and Propagation Magazine, IEEE, vol. 45, no. 3, pp. 51-82, 2003.

[12] HDMI Licensing, LLC, "High-Definition Multimedia Interface Specification Version 2.0," 2013.

[13] Grodzinsky, M., "Amendment to WFA Usage Models," IEEE 802.1109/0583ro, 2009. 